Portland State University

PDXScholar

6-6-1995

\title{
A Correlational Study: the 1-Minute Measure of Homonymy and Intelligibility
}

Tamra Leanne Day

Portland State University

Follow this and additional works at: https://pdxscholar.library.pdx.edu/open_access_etds

Part of the Speech and Rhetorical Studies Commons

Let us know how access to this document benefits you.

\section{Recommended Citation}

Day, Tamra Leanne, "A Correlational Study: the 1-Minute Measure of Homonymy and Intelligibility" (1995). Dissertations and Theses. Paper 4895.

https://doi.org/10.15760/etd.6771

This Thesis is brought to you for free and open access. It has been accepted for inclusion in Dissertations and Theses by an authorized administrator of PDXScholar. Please contact us if we can make this document more accessible: pdxscholar@pdx.edu. 


\section{THESIS APPROVAL}

The abstract and thesis of Tamra Leanne Day for the Master of Science in Speech Communication: Speech and Hearing Sciences were presented June 6,1995 , and accepted by the thesis committee and the department.

COMMITTEE APPROVALS:

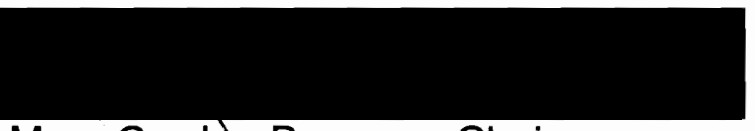

Mary Gordon-Brannan, Chair
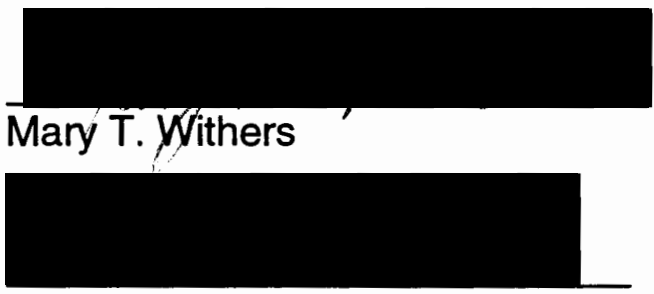

Ann Fullerton

Representative of the Office of Graduate Studies

DEPARTMENT APPROVAL:

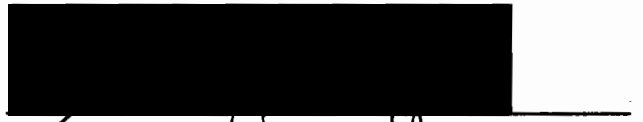

Stéphen A. Kosokoffi, Chair

Department of Speech Communication

ACCEPTED FOR PORTLAND STATE UNIVERSITY BY THE LIBRARY

by

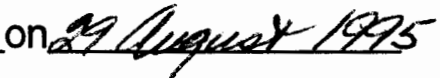


An abstract of the thesis of Tamra Leanne Day for the Master of Science in Speech Communication: Speech and Hearing Sciences presented June 6, 1995.

Title: A Correlational Study: The 1-Minute Measure of Homonymy and Intelligibility.

Identifying the severity level of unintelligibility objectively and efficiently holds critical clinical implications for speech assessment and intervention needs. The speech of children who demonstrate phonological deviations is frequently unintelligible. The use of an accurate and time-efficient measurement of intelligibility is necessary to screen children who may be producing phonological patterns that contribute to significantly reduced intelligibility in connected speech.

The purpose of this study was to investigate the degree of concurrent validity between scores received on the 1-Minute Measure of Homonymy and Intelligibility (Hodson, 1992) and speech intelligibility as measured by the percent of words understood in connected speech. For this investigation, intelligibility is operationally defined as the percent of words understood in a connected speech sample derived from orthographic transcription.

Data collected were from 48 children, aged $4: 0$ to $5: 6$, who demonstrated varying levels of phonological proficiency/deficiency. A group of four listeners who had experience treating children with phonological disorders were responsible for completing orthographic transcriptions of the 48 connected speech samples.

The two methods of assessing speech intelligibility investigated in this study were found to correlate highly $(\underline{r}=.84)$. This is considered a significant 
statistical correlation and therefore the 1-Minute Measure may be used to provide speech-language pathologists with valuable information to predict a child's intelligibility level in connected speech. A regression formula was employed to predict percentage of intelligibility when presented with a child's 1 Minute Measure score. Results from this correlational study suggest that the 1Minute Measure of Homonymy and Intelligibility may serve as an assessment tool that can provide a speech-language pathologist with some valuable information pertaining to a child's level of intelligibility in connected speech. When used with another speech assessment tool, the 1-Minute Measure may function as a screening measure to identify preschoolers who produce phonological deviations that interfere with intelligibility of conversational speech. 
A CORRELATIONAL STUDY: THE 1-MINUTE MEASURE OF HOMONYMY AND INTELLIGIBILITY.

by

TAMRA LEANNE DAY

A thesis submitted in partial fulfillment of the requirements for the degree of MASTER OF SCIENCE in SPEECH COMMUNICATION:

SPEECH AND HEARING SCIENCES

Portland State University 1995 


\section{ACKNOWLEDGMENTS}

I would like to extend my sincerest gratitude and appreciation to the members of my thesis committee, Dr. Mary Gordon-Brannan, Mary T. Withers, and Ann Fullerton. A special note of thanks is extended to Dr. Gordon-Brannan for her committed effort and valuable input throughout the duration of this project.

I extend a special thanks also to Mary T. Withers, my committee member who gave me extensive support, and reminded me to relax and take it easy every once in awhile. Ann Fullerton, the final committee member, who invested her time and energy reading and critiquing my rough drafts. I am grateful to all of my committee members for willingly taking on the task of reading my thesis in less than 3 days in preparation for my defense.

My grandmother, Betty Jo, provided me with much motivation and encouragement throughout my research experience. She always made sure that I took time to smile and to appreciate even the smallest triumph.

I dedicate this thesis to my wonderful mother, Aloa, whose love, support, and confidence made this research project possible. It was her faith that kept me going. 


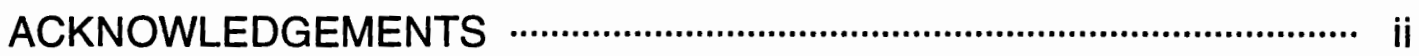

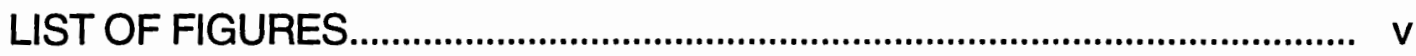

\section{CHAPTER}

I INTRODUCTION .................................................................. 1

Statement of Purpose..................................................... 2

II REVIEW OF LITERATURE

Definition of Intelligility................................................... 4

Intelligibility and Phonology.......................................... 7

Intelligibility Measurements......................................... 8

Efficiency of Assessment of Intelligibility..................... 10

Definition of Homonymy................................................. 11

III METHODS

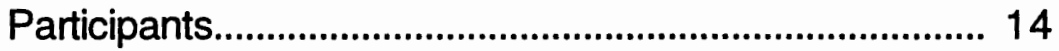

Instrumentation .............................................................. 15

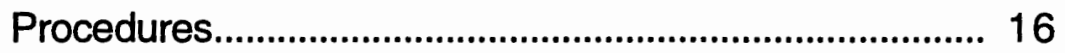

Data Analysis................................................................... 19

IV RESULTS AND DISCUSSION

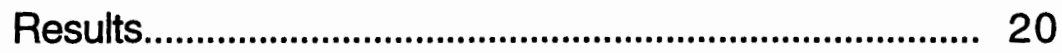

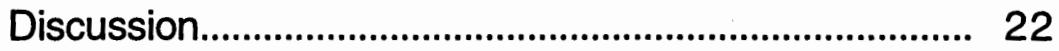


V SUMMARY AND IMPLICATIONS

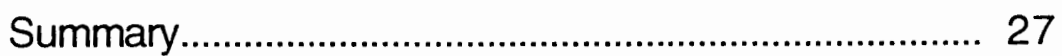

Research Implications............................................. 28

Clinical Implications.................................................... 30

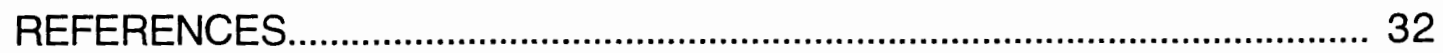
APPENDICES

A 1-MINUTE MEASURE OF HOMONYMY

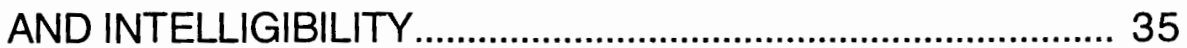

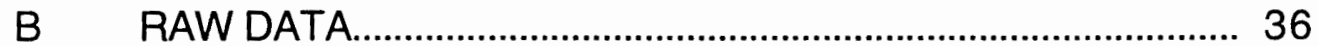




\section{LIST OF FIGURES}

FIGURE

PAGE

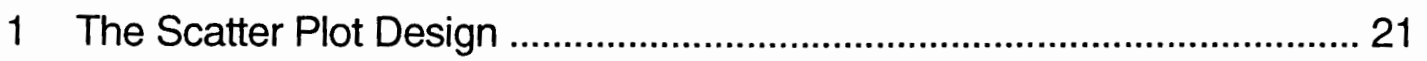




\section{CHAPTER I}

\section{INTRODUCTION AND STATEMENT OF PURPOSE}

The most practical measurement of verbal communication competence has been characterized as speech intelligibility (Metz, Samar, Schiavetti, Sitler, \& Whitehead, 1985). Determining the severity level of unintelligibility objectively and efficiently has critical clinical implications for speech assessment and intervention needs. Many speech-language clinicians assess intelligibility based on the subjective and gross measure of estimating the percentage of intelligibility in connected speech. These subjective estimations may have an effect on the nature of the child's treatment. According to Kwiatkowski and Shriberg (1992), the most valid method for measuring intelligibility is calculating the percentage of words understood from a continuous speech sample; however, this is not a time-efficient technique and therefore is seldom performed. Implications for this investigation acknowledge that most children with phonological disorders exhibit speech that is unintelligible; therefore, devising an accurate and time-efficient measurement of intelligibility is an essential element of speech assessment for these children.

The 1-Minute Measure of Homonymy and Intelligibility (1-Minute Measure) (Hodson, 1992) is an intelligibility measure composed of 15 singlesyllable words that contain the vowel $/ \mathrm{i} /$ in conjunction with later-developing consonants and consonant combinations. This test, currently in an experimental form, is designed to reflect a child's degree of intelligibility in conversational speech by eliciting the target words and then analyzing the 
speech sound deviations in terms of types of errors. A scoring system has not yet been devised for this test. Also minimal data are available to indicate whether the 1-Minute Measure can be used to predict intelligibility level as measured by percentage of words understood in connected speech samples. Based on the child's speech productions on this test, an intelligibility rating ranging from normal (adult-like) to unintelligible can be assigned.

The 1-Minute Measure may serve speech-language clinicians in a variety of ways. It is significantly more time efficient than determining the percentage of words understood in conversational speech. Perhaps this measure can function as a screening instrument to identify children whose intelligibility levels in connected speech are significantly reduced and therefore will benefit from treatment. In addition, this instrument can be used to measure the degree of change in intelligibility during intervention. The 1-Minute Measure has the capacity to be a useful tool for speech-language pathologists in a variety of ways.

\section{Statement of Purpose}

The 1-Minute Measure has been devised as a method to screen for intelligibility levels among preschool children. This correlational study was designed to determine the degree of concurrent validity between the 1-Minute Measure and speech intelligibility derived from continuous speech samples. Intelligibility, for the purpose of this investigation, is defined as the percentage of words understood in connected speech (Gordon-Brannan, 1993b). The research question addressed by this study is: is there a significant positive correlation between scores achieved on the 1-Minute Measure of Homonymy and Intelligibility and intelligibility as measured by the percent of words understood in connected speech? The corresponding null hypothesis 
investigated is: There is not a significant positive correlation between scores achieved on the 1-Minute Measure of Homonymy and Intelligibility and the percentage of words understood in connected speech. 


\section{CHAPTER $\|$}

\section{REVIEW OF LITERATURE}

A critical component for the assessment of children with phonological deficiency is accurate and efficient measurement of speech intelligibility (Gordon-Brannan, 1993a; 1993b). Measures of intelligibility provide clinicians with information necessary to determine whether intervention is needed, as well as evaluating the effectiveness of treatment strategies. Intelligibility is considered the single most practical measurement of verbal communication ability (Metz et al., 1985). Unfortunately, subjective estimations based on vague criteria are commonly used by practicing speech-language clinicians to assess intelligibility in individual clients. This type of gross assessment of intelligibility may not be reliable or valid, and may negatively affect the overall efficiency of a clinical intervention program.

It is important that measurements of intelligibility be reliable and valid because of the critical clinical component of intelligibility. Calculating the percentage of words understood in a continuous speech sample is one method of assessing intelligibility; however, this is a time-consuming and tedious process. Various research findings are suggestive of the necessity of devising intelligibility assessment tools that are reliable, valid, and time efficient.

Definition of Intelligibility

The operational definition of intelligibility varies slightly from one researcher or clinician to another. According to Bernthal and Bankson (1993), and for the purpose of this study, intelligibility is operationally defined as the 
percentage of words understood in a connected speech sample. Hodson and Paden (1991) defined intelligibility as "how well a person is understood, influenced by such factors as production of speech sounds, prosodic features, context, and listener familiarity" (p. 167).

Most definitions of intelligibility include the concept of the listener's ability to "understand" the speaker. In addition, speech intelligibility may fall on a continuum from completely intelligible to completely unintelligible (Bernthal \& Bankson, 1993). Depending on how the information is being used clinically, researchers and clinicians rely on a variety of methods to measure or quantify intelligibility. According to Gordon-Brannan (1993a), intelligibility and phonological severity levels (based on the Assessment of Phonological Processes-Revised) are highly correlated. Hodson and Paden (1991) found that the utterances typical of children with the least intelligible speech (profound phonological deficiency) are characterized by extensive omissions and some substitution errors, whereas children with slightly more intelligible speech (severe phonological deficiency) demonstrate more substitutions and fewer omissions. Yavas and Lamprecht (1988) explored the phonologies of children with moderate phonological delay and those with severe delay in terms of substitution processes and sequential processes, that is, omissions. Similar to Hodson and Paden's (1991) findings, their results support that, in children with moderate phonological delay, substitution processes dominate, and only seven kinds of omissions (cluster reduction, weak syllable deletion, final fricative deletion, final liquid deletion, intervocalic liquid deletion, and initial liquid deletion) are found; whereas, in children with severe phonological delay, a combination of substitution and sequential processes occur. In addition, according to Gordon-Brannan (1993b), children's misarticulations of particular 
sounds and sound combinations significantly reduce intelligibility in conversational speech.

Children who develop typically during the years of language acquisition are expected to increase their level of intelligibility significantly between the ages of 2:6 and 4:0. Weiss (1982) included normative data for his test of intelligibility based on continuous speech of children aged 2:6 through 4:0. $\mathrm{He}$ found that at $2: 6$, a child is $51 \%$ to $70 \%$ intelligible; at age $3: 0,71 \%$ to $80 \%$ intelligible; at $3: 6,81 \%$ to $90 \%$ intelligible; and at $4: 0,100 \%$ intelligible. Similarly, Bernthal and Bankson (1993) reported that any child older than 3 years who is unintelligible should be considered for phonological intervention. This type of normative information is critical for appropriate and accurate clinical decision-making relative to children with phonological deficiency; however, minimal normative data for speech intelligibility are available.

Identifying which techniques are most appropriate for obtaining the degree of intelligibility for children with phonological deficiencies is an area of speech-language pathology that demands further research and investigation (Gordon-Brannan, 1993a). Investigation into this area needs to focus on finding and developing efficient, reliable, and valid methods to assess overall intelligibility for children with varying degrees of phonological abilities. At the present time, many speech-language pathologists rely on impressionistic methods to estimate the percentage of intelligibility in children. This raises the issue of reliability and validity of clinical work. Gordon-Brannan (1993a) explained that there is limited research available focusing on the efficiency, reliability, and validity of methods used to assess overall intelligibility of children with varying degrees of phonological proficiency or deficiency. 
Intelligibility and Phonology

Natural phonology theorists identify strategies used by children to reduce the phonological complexity of adult language. Children will engage in systematic variations of the adult sound system throughout their language acquisition years. Such variations in the adult sound form can result in reduced speech intelligibility.

Jakobson (1968) described phonological development as an orderly progression following structural rules that are applicable no matter what language a child is acquiring. Children who have been diagnosed as demonstrating disordered phonology have phonological systems that are usually just as structured and consistent as children whose speech is developing normally (Oller, 1973). The extensive instability in childhood pronunciations suggests that a child's system includes a large number of phonological processes (Oller \& Warren, 1976). These processes often fit into strategy groups or phonetic preferences. Children, as they strive to acquire adult-like speech, will apply phonological processes that modify adult phonetic shapes, thus reducing intelligibility. Failure to account for these organizing phonological principles may lead to an inefficient treatment program (Compton, 1970).

Shriberg and Kwiatkowski (1982) applied the term natural processes to explain or account for a child's sound changes from the adult model. They explained that a child's sound change is a natural process if it meets two criteria: (a) a more complex articulatory structure at the underlying level is modified to a less complex structure at the surface level, and (b) the process widely reveals itself in other languages of the world. Natural processes theorists support the view of innate and consistent phonological processes 
occurring as children acquire language. These processes simplify the adult sound shape, therefore reducing intelligibility.

Intelligibility Measurements

Open-set word identification, closed-set word identification, and rating scale procedures are three general methods used for measuring intelligibility (Gordon-Brannan, 1993a). Open-set word identification often involves the clinician transcribing orthographically a sample and computing the percentage of words understood in continuous speech (Kent, Miolo, Bloedel., 1994; Weston \& Shriberg, 1992). Open-set word identification may also be used in single word samples, such as the first subtest of the Weiss Intelligibility Test (Weiss, 1982).

A second method used to measure intelligibility is the closed-set word identification approach that quantifies intelligibility according to words spoken from a word list. The Preschool Speech Intelligibility Measure (P-SIM) is one example of a closed-set word identification intelligibility measure. This assessment tool consists of a 50 -item intelligibility test in a multiple choice format (Wilcox, Schooling, \& Morris, 1991).

Rating scales are a third general technique used to assess intelligibility. They may be used for single word lists, paragraph reading, or continuous speech sampling. Intelligibility rating scales appear in the form of an interval scaling procedure or a direct magnitude scaling technique. Interval scaling involves listeners judging how accurately they identify the intended words of a speaker according to a predetermined scale. The predetermined scale entails a nine-point, seven-point, and five-point linear continuums. In this type of scaling, the listener assigns a number to the speech sample according to descriptors provided for the various points along the continuum or only at the 
end points of the scale. The National Technical Institute for the Deaf (NTID) Scale (Johnson, 1975) is an example of an interval scale; it uses a 5-point rating scale with descriptors assigned to each point. This procedure requires the listener to assign a number to a speech sample according to the linear continuum. The other type of scaling, direct magnitude scaling, entails placing each stimulus sample onto a continuum relative to a standard stimulus. It requires listeners to judge a speech sample "with a number that is proportional to the perceived ratios of speech intelligibility among the speech samples" as opposed to being confined to only "fixed maximum and minimum numbers at the extreme ends of the continuum" (Schiavetti, 1992, p. 20). Direct magnitude scaling therefore allows a standard to come from the upper, lower, or middle area of the rating scale. Additionally, the standard may be assigned by the investigator or it may be subjectively provided by a rater without applying a standard score.

According to Schiavetti (1992), the scaling procedure used informally provides clinicians with an estimate of the percentage of words understood by a listener. Speech-language pathologists frequently estimate the percentage of intelligibility according to known contexts and in unknown contexts. This is perhaps the most commonly used scaling procedure used by clinicians.

Although intelligibility measurement is frequently performed by using open-set word identification, closed-set word identification, and rating scales, clinicians have relied upon other approaches to assess the degree of phonological proficiency/deficiency in children. Two severity measures that have been correlated with intelligibility assessment are the phonological deviancy score (PDS) from the Assessment of Phonological Processes-Revised (Hodson, 1986), and the percentage-of-consonants correct (PCC) (Shriberg \& 
Kwiatkowski, 1982). Both have been shown to correlate with intelligibility measures (Billman, 1986; Gordon-Brannan, 1993b; Shriberg \& Kwiatkowski, 1982). In a study conducted by Garret and Moran (1992), the following phonological severity ratings were compared: (a) PDS, (b) PCC based on connected speech, (c) PCC based on single words, and (d) perceptual measures. Findings from the Garret and Moran study provide support that all five measures are highly intercorrelated. In addition, the results indicate that PCC and PDS provide similar information to the clinician in making severity assessments. These two measurements have been used at the single-word level as an assessment procedure for intelligibility. Findings from these investigations provide support for the contention that standardized single-word response tests can be valid and clinically useful tools to determine the degree of phonological proficiency/deficiency in children.

\section{Efficiency of Assessment of Intelligibility}

Some research findings have suggested that using word lists with fewer items are more time efficient and will not reduce reliability. In their original study, Tikofsky and Tikofsky (1964) used a 160-item analysis to assess and quantify the intelligibility of speech based on single words spoken by persons with dysarthria. From their results, the investigators suggested that the word list could be reduced from 160 items to a smaller set, thus increasing efficiency. Subsequently, Tikofsky (1970) reported the creation of a 50-word list constructed to serve as a basis for further studies of intelligibility. The 50 words were selected from the 160-word list following indepth item analysis. Results of this study substantiated that the original word list could be reduced to 50 words. The authors used two measures as the parameters for selection of items in the 50-word list. The two measures used were item reliability and item difficulty. 
According to the investigators of this study, these two measures were employed because they represent estimates of the discriminatory potential of each of the words used to construct the 50-item word list. This resultant word list, based on the reliability estimates of this report, indicated that the shorter list has equal, if not greater, potential for discriminating among persons with dysarthria singleword intelligibility than does the longer list.

Hodson (1992) developed The 1-Minute Measure of Homonymy and Intelligibility as a protocol to assess the intelligibility of children's speech accurately and efficiently. The 1-Minute Measure is a screening procedure consisting of 15 one-syllable words containing the vowel /i/ within a phonemic environment composed of later-developing singleton consonants and consonant sequences (Appendix A). The protocol is administered by instructing the client to repeat the words following a direct model presented by the investigator while viewing pictures that represent the words.

Definition of Homonymy

According to Weiss, Gordon, and Lillywhite (1987) and for the purpose of this investigation, homonymy will be referred to as "phonological utterances which have an excessive number of referents" (p. 109). For example, a child who produces /da/ for "dad," "dog," and "drink," is using a multiplicity of homonyms for one phonemic utterance. According to these researchers, demonstrating some homonymy during the language acquisition years is normal. These authors add that homonymy is more prevalent among children who demonstrate delayed phonological deviations.

Conclusion

Research findings have supported the idea that assessment of intelligibility must take into account the fact that the misarticulations produced 
by young children typically reveal themselves as phonological patterns, and each child's phonological system may include one or more phonological deviations. Children, as they strive to acquire adult-like speech, apply phonological processes that modify adult phonetic shapes, thus reducing intelligibility. Failure to account for these organizing phonological principles may lead to an inefficient and/or ineffective treatment program (Compton, 1970).

Knowledge of a child's phonetic preferences and consistency in misarticulations, as well as being able to identify the presence of different phonological deviations, are critical components of intelligibility assessment. In the literature, intelligibility is characterized as the single most practical measurement of oral communication ability (Metz et al., 1985). Devising accurate, objective, and time-efficient methods to assess intelligibility is critical for providing clinical implications and carrying out an appropriate and effective treatment program.

A measure designed to screen children for deficient phonological patterns indicative of inadequate speech intelligibility would save speechlanguage pathologists valuable clinic time. A review of the literature has indicated that using single-word utterances to assess the degree of phonological proficiency and/or deficiency can provide an accurate and objective measure of intelligibility in a time-efficient manner. The 1-Minute Measure of Homonymy and Intelligibility (Hodson, 1992) was designed as an intelligibility measure consisting of 15 single-syllable words containing the vowel /i/ and later developing sounds and sound combinations. This study is designed to determine whether a clinician can accurately estimate children's speech intelligibility level in connected speech based on their performance on this screening tool. If this study identifies a significant positive correlation 
between scores achieved on the 1-Minute Measure and percentage of words understood in connected speech, then this tool may be used by clinicians to screen for deficient phonological patterns in children who exemplify inadequate speech intelligibility. 


\section{CHAPTER III}

\section{METHOD}

The purpose of this study is to investigate whether a significant positive correlation exists between scores achieved on the 1-Minute Measure of Homonymy and Intelligibility (Hodson, 1992) and intelligibility as measured by the percent of words understood in connected speech samples produced by preschoolers with varying levels of phonological proficiency/deficiency. Thus, is a correlational study designed to determine the degree of concurrent validity between the 1-Minute Measure and speech intelligibility derived from connected speech samples. The data for this study were collected as a part of a larger investigation of speech intelligibility (Gordon-Brannan, 1993b). The 48 children from that study were assigned to four phonological proficiency level groups based on their performance on the 1-Minute Measure. Additionally, in the original study, a mean intelligibility percentage score was determined for each subject based on the percentage of words in a connected speech sample that were understood by a group of four listeners. Each listener was unfamiliar with the child, yet familiar with the topic of conversation, of the speech samples.

\section{Participants}

\section{Subjects}

The sample for this study participated in the original study and were comprised of 48 young children, 20 females and 28 males, ranging in age from 4:0 to $5: 6($ mean $=4: 7)$ (Gordon-Brannan, 1993b). Forty-five speakers demonstrated hearing within normal limits, and 3 had mild hearing losses with 
pure tone averages of $35 \mathrm{~dB}$ or less bilaterally classified as a slight to a mild degree of handicap (Northern \& Downs, 1991). Hearing losses with a pure tone threshold level 16-25 dB are classified as a demonstrating slight degree of handicap and those of 26-40 dB as a mild degree (Northern \& Downs, 1991). The subjects had no known motor, neurological, or physical deficiencies or laryngeal resonance deviancy that might affect speech production during the testing period. In addition, the subjects used for this study demonstrated receptive language skills appropriate for their age. The Test of Auditory Comprehension of Language-Revised (TACL-R) (Carrow-Woolfolk, 1985) was used as a screening instrument for receptive language skills.

\section{Listeners}

A group of four graduate students majoring in Speech-Language Pathology in the Portland State University Speech and Hearing Sciences Program served as listeners for the Gordon-Brannan (1993b) study. All were required to have completed coursework related to phonology and to have experience with treating children who demonstrate phonological disorders. Each listener completed orthographic transcriptions of the 48 connected speech samples.

\section{Instrumentation}

A portable Beltone pure tone audiometer, model 120, with Beltone TDH 39 headphones was used to conduct the audiometric-hearing screening. The connected speech samples and the 1-Minute Measure responses were recorded onto a different digital audiotape recorder to be later played for the listeners. The responses were then played back on the Denon digital audiotape recorder (Model DTR-80P). The 100-word connected speech samples, including both the speakers' and examiners' utterances, were dubbed 
in a random speaker order onto listener analogue tapes for later transcription. Personal analogue tape recorders were used by the listeners for orthographic transcription of the connected speech sample. Listener tapes for the 1-Minute Measure were played back on a Denon digital audiotape recorder (Model DTR80P) connected to a Sony table-top speaker (Model SRS-150).

\section{Procedures}

\section{Preliminary}

All speakers were recruited from preschools and speech-language pathology clinics or caseloads from the greater metropolitan area of Portland, Oregon. Prior to participation in the original study, the parents of each potential subject signed an informed consent form and completed a questionnaire pertaining to their child's speech, hearing, and developmental case history information.

\section{1-Minute Measure of Homonymy and Intelligibility}

The 1-Minute Measure was administered to each subject as a part of the Gordon-Brannan (1993b) study. In this screening process, the speakers repeated after the examiner 15 one-syllable words containing the vowel /i/ in conjunction with later-developing singleton consonants and consonant sequences (Appendix A). Simultaneously, pictures depicting the 15 items were shown to each subject. The productions were recorded onto digital audiotapes for later analysis and transcription. Speakers' productions were transcribed by the investigator and one of two speech-language pathology graduate students. A procedure of consensual agreement was used in order for the two transcribers to reach agreement on all phonetic transcriptions. Each phonological deviation was categorized as an omission, substitution, or distortion using the following criteria: (a) a glottal stop replacement was 
considered equivalent to an omission; (b) vowel/diphthong substitutions, as well as consonant substitutions, were considered as substitutions; (c) epenthesis of a consonant was counted as a substitution; (d) an affricate in place of an alveolar stop/strident cluster was scored as a substitution; and (e) metathesis was scored as a substitution (Gordon-Brannan, 1993a). Based on the children's performances, scores were assigned by this investigator. Collection of the Connected Speech Sample

In the Gordon-Brannan (1993b) study, a 100-word connected speech sample was collected for each subject by the investigator. The procedure began with open-ended questions and requests. Then the subjects were presented with five pictures of children involved in common activities. Finally, a picture/story book (Rylant \& Gammell, 1985) was used to elicit the speech samples. The 100-word samples were taken from the section of the speech sample involving the story book. False starts, immediate repetitions of the examiner, interjections, forms of yes and no, and filler words were not incorporated into the scored sample. The four listeners orthographically transcribed each speech sample from which a mean percentage score of words understood was derived. Before listening to the 100-word speech samples, the listeners were shown the stimuli (the book and pictures) that were used to elicit the speech sample. Each recorded speech sample was then listened to in two different listening sessions. The first listening session was in a group setting for the purpose of rating the speech intelligibility of each speaker. During the second session, the listeners listened to and transcribed the speech samples independently using their own analogue cassette tape recorders. They listened to each utterance from one to three times in their homes. 
Scoring

To score the 100-word connected speech samples, the listeners' transcriptions taken from the original study were compared to a transcription key (composed by the examiner and also by the parents of children with moderate and severe phonological deficiencies) in order to score the accuracy of orthographic transcriptions. By comparing each listener's transcriptions with the scoring key, the investigator determined the percentage of words understood by each listener for each subject. A mean percentage of words understood was determined for each speaker by averaging the percentages derived from the transcriptions completed by the four listeners.

This investigator devised a scoring system for the 1-Minute Measure. Each child was assigned a score based on the child's performance on the 1Minute Measure. The scoring system yielded scores in which the higher the score, the fewer phonological pattern errors. Each item had the potential of being worth 20 points; therefore, a test with a perfect score would receive 300 points (i.e., 15 items $X 20=300$ points). Points were subtracted for items produced with misarticulations. The following errors represented a specified point deducted from the overall score: (a) an omission resulted in losing 4 points from the total score; (b) substitutions, 3 points; and (c) distortions, 1 point. The number of points allotted to each of the possible errors was chosen based on research by Hodson and Paden (1991) and Yavas and Lamprecht (1988) who concluded that the utterances typical of children with least intelligible speech (profound) are characterized by extensive omissions and some substitutions errors, whereas children with slightly more intelligible speech (severe) demonstrate more substitutions and few omissions. 
Data Analysis

The Pearson Product Moment Correlation (Pearson- $\underline{r}$ ) was used to determine the degree of relationship between scores obtained on the 1-Minute Measure and intelligibility as measured by the percentage of words understood in a connected speech sample. A correlation coefficient of .288 at a .05 confidence level for a 48-subject study is considered statistically significant; however, for clinical purposes, a correlation of .80 to .99 beyond a .05 confidence level was considered a clinically significant correlation for this study. In order to determine the significance of this relationship, the Pearson-r value was statistically compared with the one-tailed th-test with a level of significance set at $p<.05$. In addition, the amount of shared variance between the two methods was determined by calculating $\underline{r}$-squared. MICROSOFT EXCELL was used to analyze all statistical data obtained from this study. 


\section{CHAPTER IV}

\section{RESULTS AND DISCUSSION}

\section{Results}

The purpose of this investigation was to determine the degree of relationship between the 1 - Minute Measure of Homonymy and Intelligibility and percentage of intelligibility derived from connected speech samples. The null hypothesis addressed by this study is: There is not a significant positive relationship between scores received on the 1-Minute Measure and the percentage of intelligibility achieved in connected speech samples.

The 1-Minute Measure and connected speech samples of 48 children, all aged 4:0 to 5:6, were analyzed to specify degrees of intelligibility. Raw data are presented in Appendix B (standard deviation =41.98; range =156-300). The mean for scores achieved on the 1-Minute Measure was 251.6 and the mean for intelligibility percentages was $77 \%$ (standard deviation $=18.78 \%$; range $=18.75 \%$ to $95.5 \%)$. The Pearson-r was used to investigate the research question and the corresponding null hypothesis.

The Pearson-r computation was applied to determine the strength of the relationship between the two assessment procedures for measuring intelligibility. Then a one-tailed t-test was used to determine its statistical significance. The resultant Pearson-r correlation was $.84(n=48)$. This correlation coefficient is statistically significant beyond the .05 level of confidence. Additionally, for purposes of this study, this correlation coefficient is considered to be clinically significant in that it meets the preset level of $\underline{r} \geq .80$. 
In summary, the Pearson-r computation indicates that scores from the 1-Minute Measure are highly correlated with the percentage of intelligibility derived from connected speech samples. A correlation coefficient of .84 indicates that the 1Minute Measure can account for $71 \%$ of the variability of the percentage of intelligibility, derived from connected speech, but there is still $29 \%$ of the variance unaccounted for by this tool.

The amount of covariance is visually displayed in the scatter plot design, where the 1-Minute Measure score of each subject is plotted on the $X$ axis and the percentage of intelligibility is plotted on the $Y$ axis ( shown in Figure 1). Visual inspection of the scatter plot design shows that high scores on the 1Minute Measure tend to be accompanied by high percentage of intelligibility scores on the speech samples.

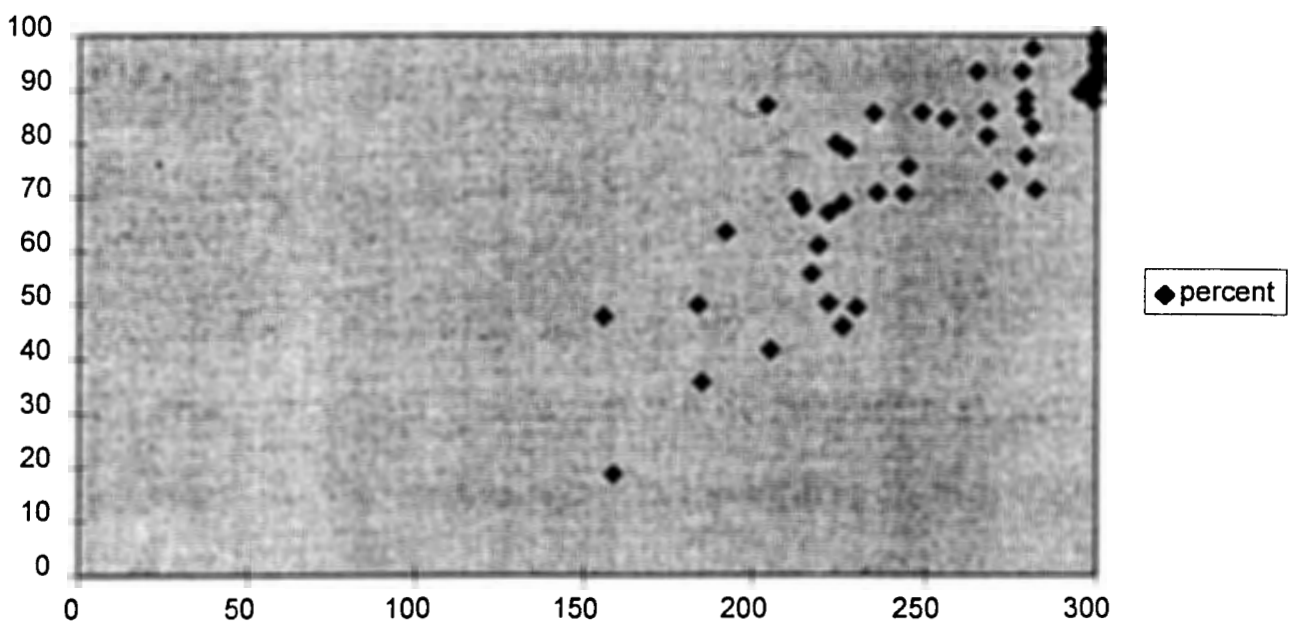

Figure 1. The scatter plot design provides a visual display of the amount of covariance between scores on the 1-Minute Measure and percentages of intelligibility from the language samples. 
A regression formula can be used to describe the estimation of one variable by another variable. The regression formula generated from the results of this study is: percentage of intelligibility $=-21+.39$ (score). This formula can be used to predict the percentage of intelligibility based on the score the child received on the 1-Minute Measure.

\section{Discussion}

Two methods of measuring speech intelligibility were correlated in this investigation: the 1-Minute Measure of Homonymy and Intelligibility and the percent of words understood in connected speech samples. The 1-Minute Measure was devised by Hodson (1992) as a method to screen for intelligibility levels among preschool children. It is currently an experimental test designed to measure a child's degree of intelligibility in connected speech by eliciting the target words and then analyzing the speech sound deviations in terms of types of errors. The later-developing sounds and sound clusters utilized in the 1Minute Measure provide the opportunity for the child to use deficient patterns. This screening tool may be more accurate than assessing intelligibility according to subjective, gross measures of estimating the percentage of intelligibility in connected speech.

Findings from this study indicate that this single-word utterance method of estimating speech intelligibility may be used as a valid and accurate screening measure. Single-word utterances that are composed of phonemic environments that provide the child with the opportunity to produce phonological deviations can give some predictive information about children's phonological patterns in connected speech and about their general intelligibility level. 
Research has provided evidence that particular phonological deviations contribute to an increase in unintelligibility (Hodson \& Paden, 1991; Yavas \& Lamprecht, 1988). Additionally, some types of errors contribute to increased unintelligibility more predominantly than others (Hodson \& Paden, 1981). Since, the words used in the 1-Minute Measure contain later developing sounds and sound clusters, the children who produce these particular words are provided with the opportunity for producing phonological deviations that will reduce overall intelligibility level in connected speech.

Weiss, Gordon, and Lillywhite (1987) explained that producing some homonymy during the preschool years is normal; however, it is more prevalent among children who demonstrate delayed phonological deviations. Based on this notion, Hodson selected 15 single words for the 1-Minute Measure, words that will provide the child with an increased chance to produce a multiplicity of homonyms as one phonemic utterance. This is the primary reason the same vowel (i.e., /i/) was used for all of the test items (Gordon-Brannan, personal communication, May 23, 1995).

The results of this study provide supporting evidence that a high correlation coefficient exists between the two measures. With a correlation coefficient of .84 , it can be inferred that as scores on the 1-Minute Measure increase, then so does the percentage of intelligibility in conversational speech (See Appendix B). This is considered a significant statistical correlation and therefore the 1-Minute Measure may be used to provide clinician's with some information useful in predicting the child's intelligibility level in connected speech.

A regression formula was generated to predict percentage of intelligibility when presented with a score received on the 1-Minute Measure. For example, 
if a child receives a score of 300 , then, according to the prediction equation, the child's intelligibility percentage will be $96 \%$. Similarly, if the child receives a score of 270 , then the predicted intelligibility level would be $84.3 \%$. A child whose score is significantly reduced, such as 200 , will yield a low intelligibility level of $77.8 \%$. This tool yields a clinically significant result, therefore by using the regression formula, a clinician can estimate intelligibility in connected speech according to the child's score on the 1-Minute Measure. In conjunction with another assessment tool, this instrument can provide practicing clinicians with valuable information regarding a child's overall intelligibility level.

The raw data indicates that children who received a perfect score of 300 on the 1-Minute Measure had intelligibility percentages of $91.5 \%$ and higher. Most children who received scores ranging from 280 to 299 on the 1-Minute Measure had intelligibility percentage of $83 \%$ and above, with the exception of one (subject 51) who received a score of $71 \%$. Most scores whether they were in the high, middle or lower ranges reflected percentages of intelligibility, accordingly. Some scores, however, did not reflect percent of words understood accurately. For example, subject number 55 received a significantly low score of 204 on the screening tool, yet was $87 \%$ intelligible to the listeners. Similarly, subject number 51 received a relatively high score on the 1-Minute Measure, however, his connected speech sample represented a low intelligibility level of $71.25 \%$

As discussed above, some subjects demonstrated significant discrepancy between the score received on the screening tool and the percent of words understood in a connected speech sample. Although $\underline{r}=.84$ indicates a strong relationship between the two tests, when the proportion of total variance is attributed to covariance (represented by squaring the correlation 
coefficient), $71 \%$ of the variance in scores between the screening tool and the connected speech samples is identified. In this study, the squared correlation coefficient of $71 \%$ can account for the variability of intelligibility, but there is still $29 \%$ that is unaccounted for by this instrument. It might be that if the scoring system accounted for homonymy in addition to deficient phonological patterns, more of the variance may be accounted for. Along these same lines, a different scoring system may result in a higher correlation and corresponding squared coefficient. For this reason, the 1-Minute Measure would be most useful as a screening tool, rather than for a diagnostic tool to measure intelligibility definitively.

The 1-Minute Measure may be more accurate than subjective estimation, and is less time consuming than collecting and transcribing a connected speech sample. The average time it takes to administer the screening tool is 1 minute and 48 seconds (see Appendix B). Thus, the 1-Minute Measure may serve as an accurate and efficient method of screening for intelligibility.

Measures of intelligibility provide speech-language pathologists with information necessary to determine whether intervention is necessary, as well as evaluating the effectiveness of treatment strategies. In addition, it is important that measurements of intelligibility be accurate and efficient because of the critical clinical implications of speech intelligibility. The traditional method of calculating the percentage of words understood in a continuous speech sample is one method of assessing intelligibility. This procedure, however, is time-consuming and tedious. Practicing clinicians are frequently challenged by time constraints, therefore collecting and transcribing a speech sample is seldom performed. Based on this correlational study, evidence is provided that the 1-Minute Measure of Homonymy and Intelligibility is an assessment tool that 
can provide a practicing speech-language pathologist with some valuable information related to intelligibility in connected speech. In conjunction with another speech assessment tool, the 1-Minute Measure may be used as a screening measure to identify children who are demonstrating phonological deviations that interfere with intelligibility of conversational speech. 


\section{CHAPTER V}

\section{SUMMARY AND IMPLICATIONS}

\section{Summary}

The 1-Minute Measure of Homonymy and Intelligibility (Hodson, 1992) is an intelligibility measure composed of 15 single-syllable words that contain the vowel /i/ in conjunction with later-developing consonants and consonant combinations. This instrument, currently in an experimental form, was designed to predict or estimate a preschooler's level of intelligibility in conversational speech by eliciting the target words and then analyzing the speech sound deviations according to types of errors. Based on the child's speech productions on this instrument, an intelligibility rating ranging from normal (adult-like) to unintelligible can be assigned.

The purpose of this study was to investigate whether a significant positive correlation exists between scores achieved on the 1-Minute Measure of Homonymy and Intelligibility and intelligibility as measured by the percent of words understood in connected speech samples produced by 4 and 5 -year old children with varying levels of phonological proficiency/deficiency. If there is a significant positive correlation between scores achieved on the 1-Minute Measure and the percentage of intelligibility in connected speech, then this method, that is more time efficient to administer, could be used with confidence as a valid instrument for screening preschoolers. 
The subjects in this study included 48 young children, 20 females and 28 males, ranging in age from 4:0 to 5:6. Each child was administered the 1Minute Measure and this score was correlated with the percentage of intelligibility ascertained from conversational speech samples. The degree of relatedness between the measures was determined using the Pearson-r. A significant correlation of .84 was found between the two protocols and the regression formula provided a prediction equation between scores achieved on the 1-Minute Measure and percentage of intelligibility in connected speech.

These statistical results support that scores received on the 1-Minute Measure can be used as a predictor of percentage of intelligibility in conversational speech for many children, and that therefore the 1-Minute Measure may be used rather than intelligibility derived from orthographic transcription of a speech sample, since it is considerably more time efficient and less tedious than collecting and transcribing a connected speech sample. However, because the 1-Minute Measure accounts for only $79 \%$ (the correlation coefficient squared) of the variance, and thus does not account for $21 \%$ of the variance of intelligibility derived from connected speech samples, it is recommended that this instrument be used as a screening tool rather than as a diagnostic instrument. It may also be useful for measuring progress throughout a treatment program.

Implications

\section{Research}

One research implication suggested by this study is the need to conduct a similar investigation on a larger group of preschool-age children to determine further the 1-Minute Measure's degree of accuracy. This study involved a group of 48 children, whereas a future research investigation should include a 
significantly increased sample of subjects. This larger pool of subjects naturally should represent a number of children at various levels of phonological proficiency/deficiency (i.e., normal, mild, moderate, and severe). Finally, the increased sample should include children who represent ages ranging from 3 to 8 years of age, as opposed to only 4 and 5 -year olds. Information ascertained from this type of investigation would provide further evidence as to the accuracy of the 1-Minute Measure.

Other possible research implications provided by this study are noteworthy. In the original investigation, the examiner used a direct model in conjunction with visual stimuli when administering the 1-Minute Measure. A future study may eliminate the examiner's model and use only the visual stimuli to elicit the subject's response. When administering the instrument in this fashion, a delayed model may be employed only when the visual stimuli does not elicit the target word. The accuracy of this instrument could, therefore, be determined by eliminating or delaying the examiner's model.

Another research implication may involve experimenting with different scoring systems. For example, subtracting additional points for homophonous word productions. The scoring system used for the purpose of this investigation entailed subtracting points for misarticulations; however, a future investigation may entail a scoring system that adds points for correct productions of target words. A future scoring system, therefore, may present itself as either more simplified or more elaborate than the one used for this study.

Finally, a future research study may integrate more of a focus on the actual production of homophonous words or sounds elicited by the 1-Minute Measure. Such an investigation may include the impact of homophonous productions on intelligibility of connected speech. Additionally, this type of 
research may lend itself to the design and implementation of a child's individualized speech treatment program.

\section{Clinical}

This correlational study provides supporting evidence that scores achieved on the 1-Minute Measure of Homonymy and Intelligibility have a significant positive correlation to percentage of intelligibility determined by a conversational speech sample. The important clinical implication provided by this study is that the 1-Minute Measure provides the clinician with valuable information regarding a child's intelligibility level in an accurate and timeefficient manner. It is common practice to assess speech intelligibility according to a conversational speech sample; however, this is a time-consuming and tedious process. In order to save time, subjective estimations based on vague criteria are commonly used by practicing speech-language clinicians to assess intelligibility, and this may have the potential to effect negatively the overall efficiency of a clinical intervention program.

According to this study, the 1-Minute Measure is a time efficient method designed to assess intelligibility accurately. The average time to administer this measure to preschool-age children is one minute and 48 seconds. The 1Minute Measure of Homonymy and Intelligibility serves to provide speechlanguage clinicians with similar information for most children regarding intelligibility level as a connected speech sample; however, it is considerably more time-efficient and systematic. Caution must be used, however, when administering the 1-Minute Measure to individual children because it may not be accurate for some children as was the case for some children in this study. It is probably most useful as a screening tool rather than as a diagnostic instrument for measuring intelligibility. It may also function as method of 
measuring progress in treatment. The instrument needs to be refined further before utilizing it as the sole measurement of intelligibility. 
References

Bernthal, J. E., \& Bankson, N. W. (1993). Articulation and phonological disorders (3rd Ed.). Englewood Cliffs, NJ: Prentice Hall.

Billman, K. S. (1986). Phonological processes and intelligibility of spontaneous utterances in young children. Unpublished master's thesis, San Diego State University, San Diego, CA.

Carrow-Woolfolk, E. (1985). Test for auditory comprehension of language-revised. Allen, TX: DLM.

Compton, A. J. (1970). Generative studies of children's phonological disorders. Journal of Speech and Hearing Disorders, 35, 315-339.

Garrett, K. K., \& Moran, M. J. (1992). A comparison of phonological measures. Language, Speech, and Hearing Services in Schools, 23, 48-51.

Gordon-Brannan, M. E., (1993a). Assessing intelligibility: Children's expressive phonologies. Topics in Language Disorders, 14, 17-25.

Gordon-Brannan, M. E., (1993b). Speech intelligibility assessment of young children with varying levels of phonological proficiency/deficiency. Doctoral dissertation, The Wichita State University, KS.

Hodson, B. W. (1986). The assessment of phonological processesrevised. Austin, TX: Pro-Ed.

Hodson, B. W.(1992) 1-minute measure of homonymy and intelligibility. Unpublished screening test. Wichita State University.

Hodson, B. W., \& Paden, E. P. (1991). Targeting intelligible speech (2nd Ed.). Austin, TX: Pro-Ed. 
Jakobson, R. (1968). Child language, aphasia and phonological universals. Transl. from the German by A. Keiler. The Hague: Mouton (originally published in 1941).

Johnson, D. D. (1975). Communication characteristics of NTID students. Journal of the Academy of Rehabilitative Audiology, $\underline{8}, 17-32$.

Kent, R. D., Miolo, G., \& Bloedel, S. (1994). The intelligibility of children's speech: A review of evaluation procedures. American Journal of SpeechLanguage Pathology: A Journal of Clinical Practice, $\underline{3}, 81$.

Kwiatkowski, J., \& Shriberg, L. D. (1992). Intelligibility assessment in developmental phonological disorders: Accuracy of caregiver gloss. Journal of Speech and Hearing Research, 35, 1095-1104.

Metz, D. E., Samar, V. J., Schiavetti, N., Sitler, R. W., \& Whitehead, R. L., (1985). Acoustic dimensions of hearing impaird speakers' intelligibility. Journal of Speech and Hearing Research, 28, 345-355.

Northern, J.L., \& Downs, M.P. (1991). Hearing in Children (4th ed.). Balthimore: Williams \& Wilkins.

Oller D. K. (1973). Regularities in abnormal child phonology. Journal of Speech and Hearing Disorders. 38 , 36-47.

Oller, D. K., \& Warren, I. (1976). On the nature of phonological capacity. Lingua, 39 , 183-189.

Rylant, C., \& Gammell, S. (1985). The relatives came. New York: Bradbury Press.

Schiavetti, N. (1992). Scaling procedures for quantification of speech, language, and hearing valuables. In R.G. Daniloff (Ed.), Articulation assessment and treatment issues. San Diego, CA: College-Hill Press. 
Shriberg, L., \& Kwiatkowski, J. (1982). Phonological disorders III: A procedure for assessing severity of involvement. Journal of Speech and Hearing Disorders, 47, 256-270.

Tikofsky, R. S., \& Tikofsky, R. P. (1964). Intelligibility measures of dysarthric speech. Journal of Speech and Hearing Research, 7, 325-333.

Tikofsky, R. S. (1970). A revised list for the estimation of dysarthric single word intelligibility. Journal of Speech and Hearing Research, 13, 59-64.

Weiss, C. E., (1982). Weiss intelligibility test. Tigard, OR: CC Publications.

Weiss, C. E., Gordon, M.E., \& Lillywhite, H.S., (1987). Clinical management of articulatory and phonological disorders. Baltimore: Williams \& Wilkins.

Weston, A. D., \& Shriberg, L. D. (1992). Contextual and linguistic correlates of intelligibility in children with developmental phonological disorders. Journal of Speech and Hearing Research, 35, 1,316-1,332.

Wilcox, K. A., Schooling, T.L., \& Morris, S.R. (1991). The preschool intelligibility measure (PSIM). Paper presented at the annual meeting of the American Speech-Language-Hearing Association, Atlanta, GA.

Yavas, M., \& Lamprecht, R. (1988). Processes and intelligibility in disordered phonology. Clinical Linguisitics and Phonoetics, $\underline{2}, 329-345$. 
1-Minute Measure of Homonymy and Intelligibility

Child's Name

Examiner

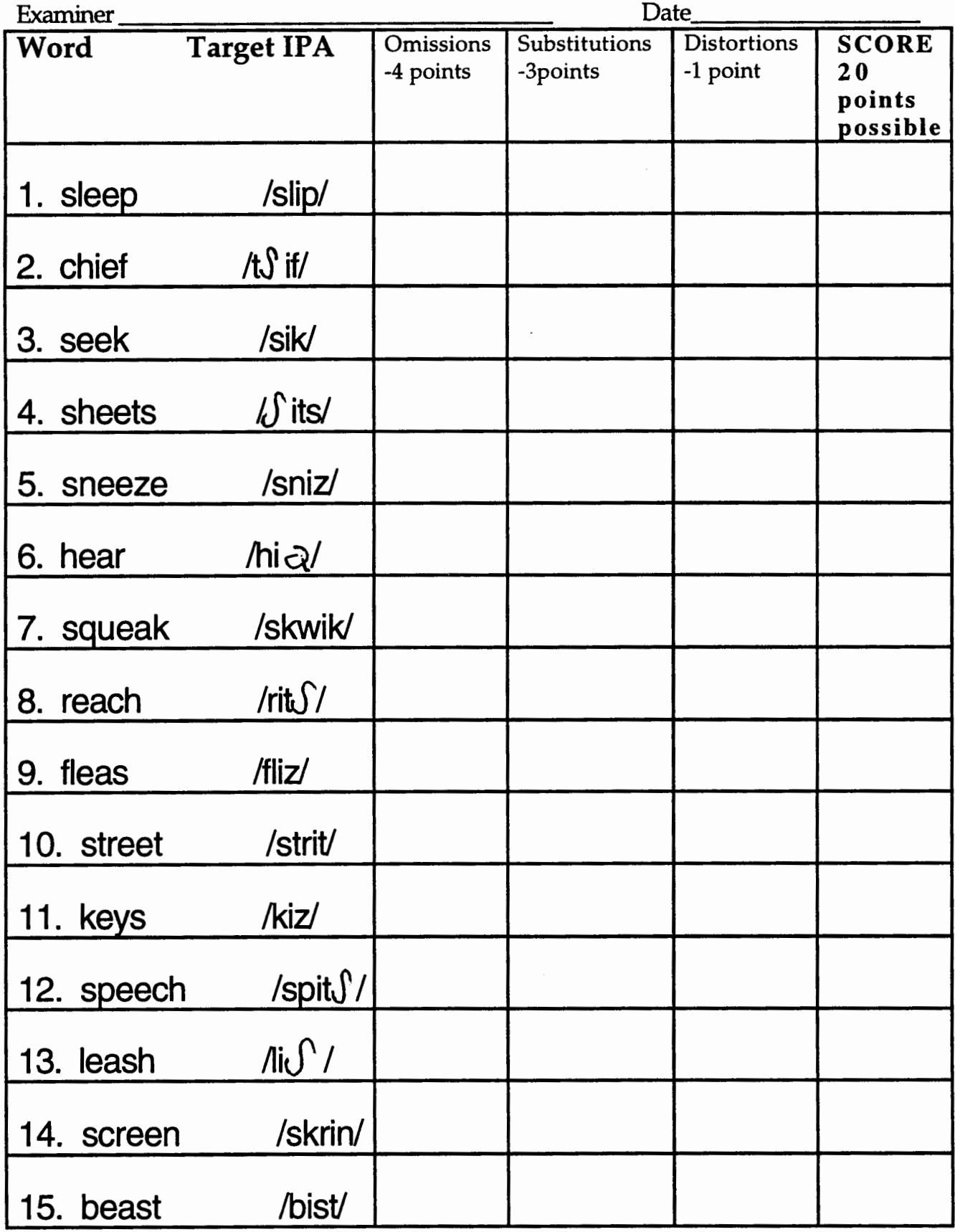

Age 
Raw Data

\begin{tabular}{|c|c|c|c|}
\hline $\begin{array}{l}\text { SCORE ON THE 1- } \\
\text { MINUTE } \\
\text { MEASURE }\end{array}$ & $\begin{array}{l}\text { PERCENT OF } \\
\text { WORDS } \\
\text { UNDERSTOOD }\end{array}$ & $\begin{array}{l}\text { AMOUNT OF TIME } \\
\text { TO ADMINISTER } \\
\text { TEST }\end{array}$ & SUBJECT NUMBER \\
\hline 300 & 99.50 & $1: 33$ & 46 \\
\hline 300 & 98.25 & $1: 39$ & 50 \\
\hline 300 & 96.25 & $1: 33$ & 38 \\
\hline 300 & 95.50 & $1: 57$ & 40 \\
\hline 300 & 95.25 & $1: 11$ & 21 \\
\hline 300 & 93.50 & $1: 40$ & 36 \\
\hline 300 & 92.00 & $1: 39$ & 32 \\
\hline 300 & 91.50 & $1: 04$ & 33 \\
\hline 299 & 90.00 & $1: 21$ & 22 \\
\hline 299 & 87.75 & $1: 40$ & 12 \\
\hline 297 & 91.25 & $2: 00$ & 47 \\
\hline 295 & 89.50 & $1: 40$ & 34 \\
\hline 282 & 71.25 & $2: 00$ & 51 \\
\hline 281 & 97.50 & $1: 43$ & 20 \\
\hline 281 & 83.00 & $1: 20$ & 10 \\
\hline 279 & 88.50 & $1: 24$ & 17 \\
\hline 279 & 86.25 & $2: 52$ & 53 \\
\hline 279 & 77.50 & $1: 49$ & 52 \\
\hline 278 & 93.25 & $1: 40$ & 4 \\
\hline 271 & 73.00 & $1: 23$ & 13 \\
\hline 268 & 86.00 & $1: 53$ & 5 \\
\hline 268 & 86.00 & $1: 43$ & 25 \\
\hline 268 & 81.25 & $1: 45$ & 42 \\
\hline 265 & 93.25 & $1: 11$ & 21 \\
\hline 256 & 84.50 & $2: 32$ & 15 \\
\hline 249 & 85.75 & $2: 06$ & 44 \\
\hline 245 & 75.50 & $2: 17$ & 41 \\
\hline 244 & 70.50 & $2: 53$ & 11 \\
\hline 236 & 70.75 & $1: 10$ & 48 \\
\hline 235 & 85.50 & $2: 08$ & 24 \\
\hline 230 & 49.50 & $2: 10$ & 23 \\
\hline 227 & 78.75 & $2: 18$ & 14 \\
\hline 226 & 68.75 & $1: 55$ & 1 \\
\hline 224 & 80.00 & $1: 46$ & 7 \\
\hline 225 & 46.00 & 2:00 & 43 \\
\hline 222 & 67.00 & $1: 28$ & 27 \\
\hline
\end{tabular}




\begin{tabular}{|l|}
\hline 222 \\
\hline 219 \\
\hline 217 \\
\hline 214 \\
\hline 213 \\
\hline 205 \\
\hline 204 \\
\hline 192 \\
\hline 185 \\
\hline 184 \\
\hline 159 \\
\hline 156 \\
\hline
\end{tabular}

50.25

61.00

55.75

68.00

69.50

41.75

87.00

63.50

35.75

50.00

18.74

47.75
$1: 30$

2:20

$1: 39$

2:20

$1: 30$

2:30

$1: 23$

2:03

2:30

$1: 40$

$1: 58$

$1: 30$

\section{8}

16

49

2

9

6

55

18

3

45

28

56 